\title{
Symplectic map of crab cavity
}

\author{
Hung Jin Kim \\ Fermi National Accelerator Laboratory, Batavia, Illinois 60510, USA \\ hjkim@fnal.gov
}

\begin{abstract}
In this paper two types of crab cavity are introduced, which are TM and TEM mode cavities. The equation of motion of both cavities is obtained from Lorentz force and Hamiltonian respectively. The equation of motion is solved using thin-lens approximation.
\end{abstract}

\section{CRAB CAVITY}

Consider electromagnetic waves confined to the interior of a hollow pipe, or wave guide. We assume that the wave guide is a perfect conductor, so that $\mathbf{E}=0$ and $\mathbf{B}=0$ inside the material itself, and hence the boundary conditions at the inner wall are

$$
\mathbf{E}_{\|}=0, \quad \mathbf{B}_{\perp}=0 .
$$

We are interested in monochromatic waves that propagate down the tube, so $\vec{E}$ and $\vec{B}$ have the generic form

$$
\begin{aligned}
\tilde{\mathbf{E}}(x, y, z, t) & =\tilde{\mathbf{E}}_{0}(x, y) e^{i(k z-\omega t),} \\
\tilde{\mathbf{B}}(x, y, z, t) & =\tilde{\mathbf{B}}_{0}(x, y) e^{i(k z-\omega t)},
\end{aligned}
$$

The electric and magnetic fields must satisfy Maxwell's equations in the interior of the wave guide:

$$
\begin{array}{ll}
\nabla \cdot \mathbf{E}=0, & \nabla \times \mathbf{E}=-\frac{\partial \mathbf{B}}{\partial t}, \\
\nabla \cdot \mathbf{B}=0, & \nabla \times \mathbf{B}=\frac{1}{c^{2}} \frac{\partial \mathbf{E}}{\partial t} .
\end{array}
$$

The problem is to find functions $\tilde{\mathbf{E}}_{0}$ and $\tilde{\mathbf{B}}_{0}$ such that the fields, Eq. (2), obey the differential equations, Eq. (3), subject to boundary conditions, Eq. (1). It suffices to determine the longitudinal components $E_{z}$ and $B_{z}$; if we know those, we could quickly calculate all the others, just by differentiating. If $E_{z}=0$ we call these TE ("transverse electric") waves; if $B_{z}=0$ they are called TM ("transverse magnetic") waves; if both $E_{z}=0$ and $B_{z}=0$, we call them TEM ("transverse electromagnetic") waves.

\section{A. TM mode cavity}

The TM110 mode in a cylindrical geometry is shown in Fig. 1. The electromagnetic field of TM110 mode in a cylindrical

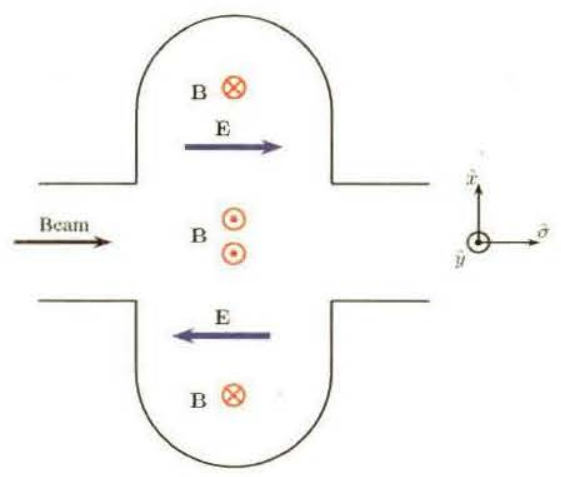

Figure 1: TM110 mode in a cylindrical geometry. There is no electric field along $z$-axis, but only vertical magnetic field appears. The vertical magnetic field gives a deflecting kick. 


\title{
Symplectic map of crab cavity
}

\author{
Hung Jin Kim \\ Fermi National Accelerator Laboratory, Batavia, Illinois 60510, USA \\ hjkim@fnal.gov
}

\begin{abstract}
In this paper two types of crab cavity are introduced, which are TM and TEM mode cavities. The equation of motion of both cavities is obtained from Lorentz force and Hamiltonian respectively. The equation of motion is solved using thin-lens approximation.
\end{abstract}

\section{CRAB CAVITY}

Consider electromagnetic waves confined to the interior of a hollow pipe, or wave guide. We assume that the wave guide is a perfect conductor, so that $\mathbf{E}=0$ and $\mathbf{B}=0$ inside the material itself, and hence the boundary conditions at the inner wall are

$$
\mathbf{E}_{\|}=0, \quad \mathbf{B}_{\perp}=0 .
$$

We are interested in monochromatic waves that propagate down the tube, so $\vec{E}$ and $\vec{B}$ have the generic form

$$
\begin{aligned}
\tilde{\mathbf{E}}(x, y, z, t) & =\tilde{\mathbf{E}}_{0}(x, y) e^{i(k z-\omega t),} \\
\tilde{\mathbf{B}}(x, y, z, t) & =\tilde{\mathbf{B}}_{0}(x, y) e^{i(k z-\omega t)},
\end{aligned}
$$

The electric and magnetic fields must satisfy Maxwell's equations in the interior of the wave guide:

$$
\begin{array}{ll}
\nabla \cdot \mathbf{E}=0, & \nabla \times \mathbf{E}=-\frac{\partial \mathbf{B}}{\partial t}, \\
\nabla \cdot \mathbf{B}=0, & \nabla \times \mathbf{B}=\frac{1}{c^{2}} \frac{\partial \mathbf{E}}{\partial t} .
\end{array}
$$

The problem is to find functions $\tilde{\mathbf{E}}_{0}$ and $\tilde{\mathbf{B}}_{0}$ such that the fields, Eq. (2), obey the differential equations, Eq. (3), subject to boundary conditions, Eq. (1). It suffices to determine the longitudinal components $E_{z}$ and $B_{z}$; if we know those, we could quickly calculate all the others, just by differentiating. If $E_{z}=0$ we call these TE ("transverse electric") waves; if $B_{z}=0$ they are called TM ("transverse magnetic") waves; if both $E_{z}=0$ and $B_{z}=0$, we call them TEM ("transverse electromagnetic") waves.

\section{A. TM mode cavity}

The TM110 mode in a cylindrical geometry is shown in Fig. 1. The electromagnetic field of TM110 mode in a cylindrical

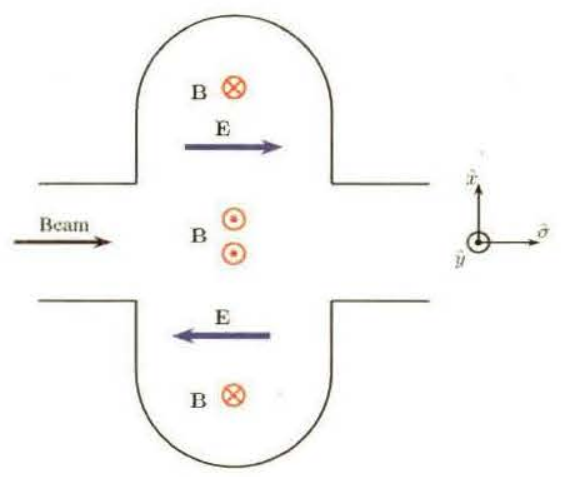

Figure 1: TM110 mode in a cylindrical geometry. There is no electric field along $z$-axis, but only vertical magnetic field appears. The vertical magnetic field gives a deflecting kick. 
The time transit factor $T$ arises from the fact that a particle passes through the rf gap within a finite time interval so that the energy gain is the time average of the electric field in the gap during the transit time. Since the transverse beam size is much smaller than the cavity diameter, i.e., $\frac{r}{R} \ll 1$, the Bessel function can be expanded as

$$
\begin{aligned}
& J_{\alpha}(x)=\sum_{m=0}^{\infty} \frac{(-1)^{m}}{m ! \Gamma(m+\alpha+1)}\left(\frac{1}{2} x\right)^{2 m+\alpha}, \\
& J_{1}(x)=\frac{1}{2} x-\frac{1}{16} x^{3}+\frac{1}{192} x^{5} \cdots, \\
& J_{2}(x)=\frac{1}{8} x^{2}-\frac{1}{96} x^{4}+\cdots,
\end{aligned}
$$

where $\Gamma(n)=(n-1)$ !. Then Eq. (8) can be approximated for small amplitude beam as follows

$$
\begin{aligned}
& \Delta p_{x}=\frac{q \tilde{V}_{c c}}{E}\left[1+\frac{1}{8}\left(\frac{\alpha}{R}\right)^{2}\left(x^{2}-y^{2}\right)-\frac{1}{96}\left(\frac{\alpha}{R}\right)^{4}\left(x^{4}-y^{4}\right)+\cdots\right] \sin \frac{\omega z}{\beta c}, \\
& \Delta p_{y}=\frac{q \tilde{V}_{c c}}{E}\left[-\frac{1}{4}\left(\frac{\alpha}{R}\right)^{2}+\frac{1}{24}\left(\frac{\alpha}{R}\right)^{4} y^{2}+\cdots\right] x y \sin \frac{\omega z}{\beta c}, \\
& \Delta p_{s}=\frac{q \tilde{V}_{c c}}{E}\left[1-\frac{1}{8}\left(\frac{\alpha}{R}\right)^{2}\left(-\frac{1}{3} x^{2}+y^{2}\right)+\frac{1}{96}\left(\frac{\alpha}{R}\right)^{4}\left(-\frac{1}{5} x^{4}+y^{4}\right)+\cdots\right] \frac{\omega}{\beta c} x \cos \frac{\omega z}{\beta c} .
\end{aligned}
$$

In order to make the approximate solution be symplectic, Eq. (10) is rearranged in higher order terms. Note that the radius of KEK crab cavity is $R=0.433 \mathrm{~m}$ and $\alpha=3.832$. At the (proposed) crab cavity location of SPS, the rms beam size is $\sigma_{x}=0.91 \mathrm{~mm}$.

As a check, let's see the equation of motion of particles whose transverse amplitude is small. At coordinates $(x, y, s)=$ $(0,0, s)$, the electromagnetic fields are given by

$$
\begin{aligned}
E_{x} & =E_{y}=B_{x}=B_{s}=0, \\
B_{y} & =\frac{1}{2 c} \mathcal{E}_{0} \sin (\omega t), \\
E_{s} & =\frac{\mathcal{E}_{0}}{2} \frac{\omega}{c} x \cos (\omega t) .
\end{aligned}
$$

Note that $B_{r}$ and $B_{\phi}$ give the vertical magnetic field at $r=0$. Then one can get the equation of motion:

$$
\begin{aligned}
\frac{d p_{x}}{d t} & =-\frac{q}{2 p_{0} c} \mathcal{E}_{0} \beta c \sin \left(\omega\left(t-\frac{1}{\beta c} z\right)\right), \\
\frac{d p_{y}}{d t} & =0, \\
\frac{d p_{s}}{d t} & =\frac{q \mathcal{E}_{0}}{2 p_{0} c} \omega x \cos \left(\omega\left(t-\frac{1}{\beta c} z\right)\right) .
\end{aligned}
$$

As above, it is assumed that the cavity field is synchronized with the beam bunch, i.e., a synchronous particle sees the sine wave without a phase. The $z$ is the longitudinal distance from the synchronous particle. The reference particle passes through the cavity gap in time $t \in n T_{0}+(-\lambda / 2 \beta c, \lambda / 2 \beta c)$, where $\lambda$ is the cavity gap width. The cavity kick for a particle per passage is

$$
\begin{aligned}
\Delta p_{x} & =-\frac{q}{2 p_{0} c} \mathcal{E}_{0} \beta c \int_{-\lambda / 2 \beta c}^{\lambda / 2 \beta c} \sin \left(\omega\left(t-\frac{1}{\beta c} z\right)\right) d t, \\
& =\frac{q}{p_{0} c} \mathcal{E}_{0} \frac{\beta c}{\omega} \sin \left(\frac{\omega \lambda}{2 \beta c}\right) \sin \frac{\omega z}{\beta c}, \\
\Delta p_{y} & =0, \\
\Delta p_{s} & =\frac{q \mathcal{E}_{0}}{p_{0} c} \sin \frac{\omega \lambda}{2 \beta c} x \cos \frac{\omega z}{\beta c} .
\end{aligned}
$$

Here $k=\omega / c$. Equation (13) can be rewritten by

$$
\begin{aligned}
& \Delta p_{x}=\frac{q V_{c c}}{E} \sin \frac{\omega z}{\beta c} \\
& \Delta p_{y}=0, \\
& \Delta p_{s}=\frac{q V_{c c}}{E} \frac{\omega}{\beta c} x \cos \frac{\omega z}{\beta c},
\end{aligned}
$$

where the beam energy $E$ is $E=p_{0} c$, the cavity energy $q V_{c c}=q \mathcal{E}_{0} \lambda T$, the transit time factor $T=\sin \left(\frac{\omega \lambda}{2 \beta c}\right) /\left(\frac{\omega \lambda}{2 \beta c}\right)$ 


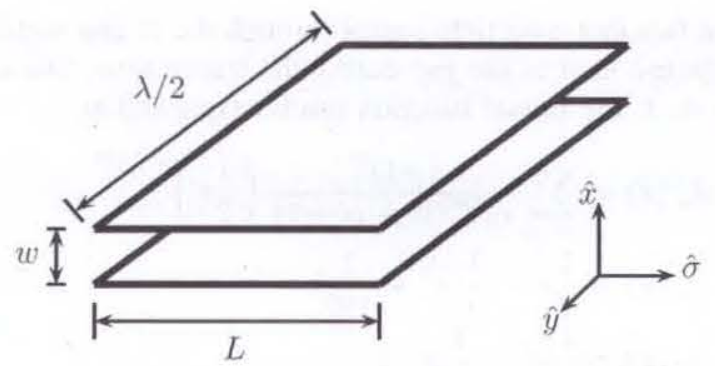

Figure 2: Parallel plate waveguide. The beam direction is along $\hat{\sigma}$.

1) Symplecticity: The Jacobian matrix resulting from Eq. (8) takes the form

$$
\begin{aligned}
\mathcal{J} & =\frac{\partial\left(x^{f}, p_{x}^{f}, y^{f}, p_{y}^{f}, z^{f}, p_{z}^{f}\right)}{\partial\left(x^{i}, p_{x}^{i}, y^{i}, p_{y}^{i}, z^{i}, p_{z}^{i}\right)} \\
& =\left(\begin{array}{cccccc}
1 & 0 & 0 & 0 & 0 & 0 \\
j_{21} & 1 & j_{23} & 0 & j_{25} & 0 \\
0 & 0 & 1 & 0 & 0 & 0 \\
j_{41} & 0 & j_{43} & 1 & j_{45} & 0 \\
0 & 0 & 0 & 0 & 1 & 0 \\
k_{61} & 0 & j_{63} & 0 & j_{65} & 1
\end{array}\right),
\end{aligned}
$$

where the elements of Jacobian matrix are given by

$$
\begin{gathered}
j_{21}=\frac{\partial p_{x}^{f}}{\partial x}=\cdots \\
\ldots \\
j_{65}=\frac{\partial p_{z}^{f}}{\partial z}=\cdots
\end{gathered}
$$

For a symplecticity condition, the Jacobian should satisfy

$$
\mathcal{J}^{T} \mathcal{S} \mathcal{J}=\mathcal{S}
$$

Here the matrix $\mathcal{S}$ is given by

$$
\mathcal{S}=\left(\begin{array}{ccc}
\mathcal{S}_{2} & 0 & 0 \\
0 & \mathcal{S}_{2} & 0 \\
0 & 0 & \mathcal{S}_{2}
\end{array}\right), \quad \mathcal{S}_{2}=\left(\begin{array}{cc}
0 & -1 \\
+1 & 0
\end{array}\right)
$$

\section{B. TEM mode cavity}

1) Parallel plate: Equation of motion from Lorentz force: The wave guide of parallel plate gives a TEM mode wave, as shown in Fig. 2. The electric and magnetic field of TEM waveguide in parallel plate are given by

$$
\begin{aligned}
& \mathbf{E}(x, y, \sigma, t)=\hat{x} \mathcal{E}_{0} \cos \left(\frac{2 \pi y}{\lambda}\right) \sin (\omega t), \\
& \mathbf{B}(x, y, \sigma, t)=\hat{\sigma} \frac{\mathcal{E}_{0}}{Z_{0}} \sin \left(\frac{2 \pi y}{\lambda}\right) \cos (\omega t) .
\end{aligned}
$$

Note that the TEM wave propagates along the $y$ direction. With application of $d \mathbf{p} / d t=\frac{1}{p_{0}} q(\mathbf{E}+\mathbf{v} \times \mathbf{B})$, the equation of motion is given by

$$
\frac{d p_{x}}{d t}=\frac{q \mathcal{E}_{0}}{p_{0}} \cos (k y) \sin (\omega t), \quad \frac{d p_{y}}{d t}=0, \quad \frac{d p_{s}}{d t}=0 .
$$

Here it is assumed that the cavity field is synchronized with the beam bunch, i.e., a synchronous particle sees the sine wave without a phase. The $z$ is the longitudinal distance from the synchronous particle. The off-momentum particles see the wave as $\sin (\omega(t-z / \beta c))$. We assume that the reference particle passes through the cavity gap in time $t \in n T_{0}+(-L / 2 \beta c, L / 2 \beta c)$, where $L$ is the cavity gap width. Then we can get a cavity kick for a particle per passage,

$$
\Delta p_{x}=-\frac{q V_{c c}}{E} \cos (k y) \sin \frac{\omega z}{\beta c}, \quad \Delta p_{y}=0, \quad \Delta p_{s}=0,
$$


where the beam energy $E$ is $E=p_{0} \beta c$, the cavity energy $q V_{c c}=q \mathcal{E}_{0} \lambda T$, the transit time factor $T=\sin \left(\frac{\omega L}{2 \beta c}\right) /\left(\frac{\omega L}{2 \beta c}\right)$. In order to verify the symplecticity condition of Eq. (17), one can get the Jacobian

$$
\begin{aligned}
\mathcal{J} & =\frac{\partial\left(x^{f}, p_{x}^{f}, y^{f}, p_{y}^{f}, z^{f}, p_{z}^{f}\right)}{\partial\left(x^{i}, p_{x}^{i}, y^{i}, p_{y}^{i}, z^{i}, p_{z}^{i}\right)} \\
& =\left(\begin{array}{ccccccc}
1 & 0 & 0 & 0 & 0 & 0 \\
0 & 1 & j_{23} & 0 & j_{25} & 0 \\
0 & 0 & 1 & 0 & 0 & 0 \\
0 & 0 & 0 & 1 & 0 & 0 \\
0 & 0 & 0 & 0 & 1 & 0 \\
0 & 0 & 0 & 0 & 0 & 1
\end{array}\right),
\end{aligned}
$$

where $j_{23}=\frac{q V_{c c}}{E} k \sin (k y) \sin \left(\frac{\omega z}{\beta c}\right)$ and $j_{25}=-\frac{q V_{c c}}{E} \frac{\omega}{\beta c} \cos (k y) \cos \frac{\omega z}{\beta c}$. Then one can get

$$
\mathcal{J}^{T} \mathcal{S} \mathcal{J}-\mathcal{S}=\left(\begin{array}{cccccc}
0 & 0 & j_{23} & 0 & j_{25} & 0 \\
0 & 0 & 0 & 0 & 0 & 0 \\
-j_{23} & 0 & 0 & 0 & 0 & 0 \\
0 & 0 & 0 & 0 & 0 & 0 \\
-j_{25} & 0 & 0 & 0 & 0 & 0 \\
0 & 0 & 0 & 0 & 0 & 0
\end{array}\right)
$$

The equation of motion in terms of mechanical momentum does not satisfy the symplecticity condition. However, the symplecticity condition is satisfied in the canonical coordinates, because the horizontal canonical momentum $\Delta p_{x}=0$ (see Eq. (25)).

Equation of motion from Hamiltonian: Let us consider the Hamiltonian to derive the equation of motion in the parallel plate of TEM mode. In the TEM mode of parallel plate, the vector potential experienced by a test particle moving with a velocity $\beta c$ is

$$
\mathbf{A}(x, y, z ; s)=\hat{x} A_{x}(y, t)=\hat{x} \frac{\mathcal{E}_{0}}{\omega} \cos \left(\frac{2 \pi y}{\lambda}\right) \cos \left(\frac{\omega}{\beta c}(s-z)\right) .
$$

The Hamiltonian of TEM mode cavity is given by

$$
\mathcal{H}=\frac{\left(p_{x}-\frac{q}{p_{0}} A_{x}\right)^{2}+p_{y}^{2}}{2\left(1+f\left(p_{z}\right)\right)}+p_{z}-f\left(p_{z}\right) \text {. }
$$

Canonical transformation is applied in order to remove the vector potential term in $\left(p_{x}-\frac{q}{p_{0}} A_{x}\right)^{2}$. The transformation is made via a (type 3$)$ generating function $G\left(\bar{x}, \bar{y}, \bar{z}, p_{x}, p_{y}, p_{z} ; s\right)$ :

$$
\begin{aligned}
G\left(\bar{x}, \bar{y}, \bar{z}, p_{x}, p_{y}, p_{z} ; s\right) & =-\bar{x} p_{x}-\bar{y} p_{y}-\bar{z} p_{z}+\bar{x} \frac{q}{p_{0}} A_{x}, \\
& =-\bar{x} p_{x}-\bar{y} p_{y}-\bar{z} p_{z}+\bar{x} \frac{q \mathcal{E}_{0}}{p_{0} \omega} \cos (k y) \cos \left(\frac{\omega}{\beta c}(s-z)\right) .
\end{aligned}
$$

The generating function gives

$$
\begin{array}{ll}
x=-\frac{\partial G}{\partial p_{x}}=\bar{x}, & p_{\bar{x}}=-\frac{\partial G}{\partial \bar{x}}=p_{x}-\frac{q}{p_{0}} A_{x}, \\
y=-\frac{\partial G}{\partial p_{y}}=\bar{y}, & p_{\bar{y}}=-\frac{\partial G}{\partial \bar{y}}=p_{z}, \\
z=-\frac{\partial G}{\partial p_{z}}=\bar{z}, & p_{\bar{z}}=-\frac{\partial G}{\partial \bar{z}}=p_{z} .
\end{array}
$$

Note that $p_{\bar{x}}, p_{\bar{y}}$, and $p_{\bar{z}}$ are the mechanical momentum. New Hamiltonian $\overline{\mathcal{H}}$ is given by

$$
\begin{aligned}
\overline{\mathcal{H}} & =\mathcal{H}+\frac{\partial G}{\partial s}, \\
& =\frac{p_{\bar{x}}^{2}+p_{\bar{y}}^{2}}{2\left(1+f\left(p_{\bar{z}}\right)\right)}+p_{\bar{z}}-f\left(p_{\bar{z}}\right)-\frac{q \mathcal{E}_{0}}{p_{0} \beta c} \bar{x} \cos (k y) \sin \left(\frac{\omega}{\beta c}(s-z)\right) .
\end{aligned}
$$

The new Hamiltonian is

$$
\mathcal{H}=\frac{1}{2} \frac{p_{\bar{x}}^{2}+p_{\bar{y}}^{2}}{1+f\left(p_{\bar{z}}\right)}+p_{\bar{z}}-f\left(p_{\bar{z}}\right)-\frac{q \mathcal{E}_{0}}{p_{0} \beta c} \bar{x} \cos (k y) \sin \left(\frac{\omega}{\beta c}(s-z)\right) .
$$



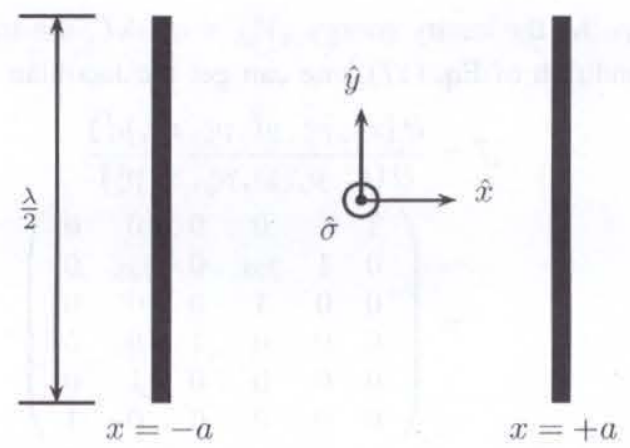

Figure 3: TEM mode waveguide of parallel two lines. The beam direction is along $\hat{\sigma}$.

Then one can get the equation of motion using Eq. (47)

$$
\begin{aligned}
\frac{d}{d s} \bar{x} & =+\frac{\partial \mathcal{H}}{\partial p_{\bar{x}}}=\frac{p_{\bar{x}}}{1+f\left(p_{\bar{z}}\right)}, \\
\frac{d}{d s} p_{\bar{x}} & =-\frac{\partial \mathcal{H}}{\partial \bar{x}}=\frac{q \mathcal{E}_{0}}{p_{0} \beta c} \cos (k y) \sin \left(\frac{\omega}{\beta c}(s-z)\right), \\
\frac{d}{d s} \bar{y} & =+\frac{\partial \mathcal{H}}{\partial p_{\bar{y}}}=\frac{p_{\bar{y}}}{1+f\left(p_{\bar{z}}\right)}, \\
\frac{d}{d s} p_{\bar{y}} & =-\frac{\partial \mathcal{H}}{\partial \bar{y}}=0, \\
\frac{d}{d s} \bar{z} & =+\frac{\partial \mathcal{H}}{\partial p_{\bar{z}}}=1-\left(1+\frac{p_{\bar{x}}^{2}+p_{\bar{y}}^{2}}{2\left(1+f\left(p_{\bar{z}}\right)\right)^{2}}\right) f^{\prime}\left(p_{\bar{z}}\right), \\
\frac{d}{d s} p_{\bar{z}} & =-\frac{\partial \mathcal{H}}{\partial \bar{z}}=0 .
\end{aligned}
$$

Using the thin-lens approximation of the cavity, one can get the solution of Eq. (24):

$$
\begin{array}{rlrl}
\Delta \bar{x} & =0, & & \Delta p_{\bar{x}}=-\frac{q V_{c c}}{E} \cos (k y) \sin \left(\frac{\omega z}{\beta c}\right), \\
\Delta \bar{y}=0, & \Delta p_{\bar{y}}=0, \\
\Delta \bar{z}=0, & \Delta p_{\bar{z}}=0,
\end{array}
$$

where the beam energy $E$ is $E=p_{0} \beta c$, the cavity energy $q V_{c c}=q \mathcal{E}_{0} \lambda T$, the transit time factor $T=\sin \left(\frac{\omega L}{2 \beta c}\right) /\left(\frac{\omega L}{2 \beta c}\right)$. Equation (25) is the same as Eq. (17). Note that $\Delta p_{x}=A_{x}^{f}-A_{x}^{i}-\frac{q V_{c c}}{E} \cos (k y) \sin \left(\frac{\omega z}{\beta c}\right)=0$.

2) Parallel wire: The electric and magnetic fields in a TEM resonant structure are

$$
\begin{aligned}
& \mathbf{E}(x, y, \sigma, t)=\mathbf{E}(x, \sigma) \cos \left(\frac{2 \pi y}{\lambda}\right) \sin (\omega t), \\
& \mathbf{B}(x, y, \sigma, t)=\frac{\mathbf{E}(x, \sigma)}{Z_{0}} \times \hat{y} \sin \left(\frac{2 \pi y}{\lambda}\right) \cos (\omega t) .
\end{aligned}
$$

Here $Z_{0}=\frac{\lambda \omega}{2 \pi}=\frac{\omega}{k}=\sqrt{\epsilon_{0} \mu_{0}}=1 / c$. Note that temporal term in Eq. (26) is different from [1]. cos $\omega t$ gives the deflecting kick, i.e., the amplitude of kicks is maximum at the longitudinal center of the beam. It is assumed that two infinitely long lines are parallel to the $y$-axis, and crossing the $(x, \sigma)$ plane at $x= \pm a, \sigma=0$, and carrying uniform linear charge per unit length $q$, as shown in Fig. 4. Note that the TEM wave propagates along the $y$ direction. The potential is given by

$$
V(x, \sigma)=\frac{q}{4 \pi \epsilon_{0}} \ln \left(\frac{r_{-}^{2}}{r_{+}^{2}}\right),
$$

where

$$
r_{-}^{2}=(x-a)^{2}+\sigma^{2}, \quad r_{+}^{2}=(x+a)^{2}+\sigma^{2} .
$$




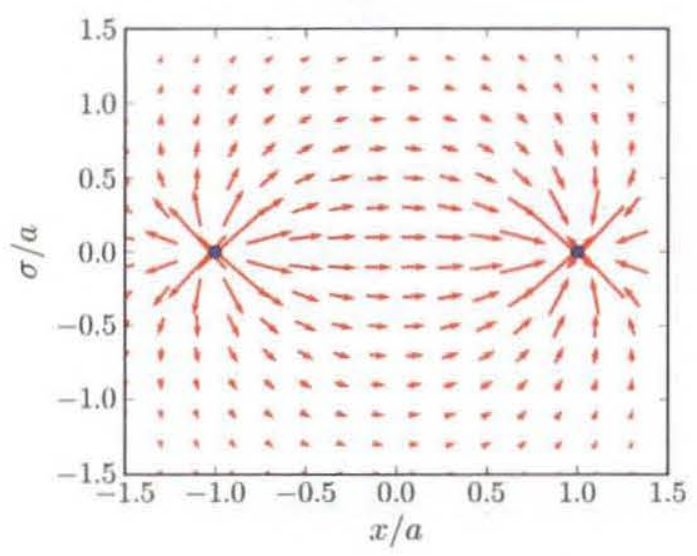

(a)

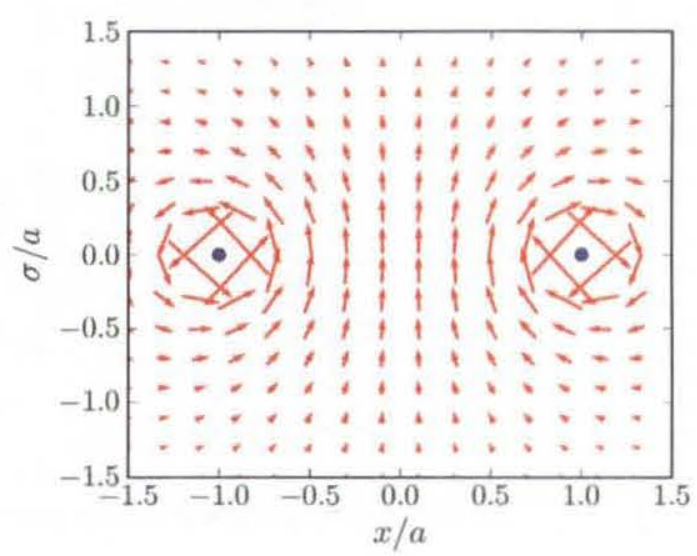

(b)

Figure 4: Transverse electromagnetic field of TEM mode cavity: (a) electric field, and (b) magnetic field. The two lines are located at $(x, y)=( \pm a, 0)$.

The electric field is

$$
\begin{aligned}
& E_{x}(x, \sigma)=-\frac{\partial V}{\partial x}=-\frac{a q}{\pi \epsilon_{0}}\left[\frac{x^{2}-a^{2}-\sigma^{2}}{r_{-}^{2} r_{+}^{2}}\right], \\
& E_{\sigma}(x, \sigma)=-\frac{\partial V}{\partial \sigma}=-\frac{a q}{\pi \epsilon_{0}}\left[\frac{2 x \sigma}{r_{-}^{2} r_{+}^{2}}\right] .
\end{aligned}
$$

Figure 4 shows the transverse electromagnetic field of TEM mode cavity.

With application of $d \mathbf{p} / d t=\frac{1}{p_{0}} q(\mathbf{E}+\mathbf{v} \times \mathbf{B})$ and $\mathbf{v}=\beta c \hat{\sigma}$, the equation of motion is

$$
\begin{aligned}
\frac{d p_{x}}{d t} & =\frac{q}{p_{0}} E_{x}(x, \sigma) \cos (k y) \sin (\omega t), \\
\frac{d p_{y}}{d t} & =-\frac{q}{p_{0}} \frac{\beta c}{Z_{0}} E_{\sigma}(x, \sigma) \sin (k y) \cos (\omega t), \\
\frac{d p_{z}}{d t} & =\frac{q}{p_{0}} E_{\sigma}(x, \sigma) \cos (k y) \sin (\omega t) .
\end{aligned}
$$

Note that $\mathbf{p}$ is normalized by $p_{0}$. The horizontal electric field $E_{x}$ has a maximum value at the origin $(x, \sigma)=(0,0)$. The vertical electric field $E_{\sigma}$ has a minimum at the origin. Momentum changes, $\Delta p_{y}$ and $\Delta p_{s}$, are expected to be small or negligible, compared to horizontal momentum change. At small horizontal displacement, i.e., $x \simeq 0$, the horizontal electric field is $E_{x}(0, \sigma)=\frac{a q}{\pi \epsilon_{0}} \frac{1}{a^{2}+\sigma^{2}}$. As a test particle passes through the cavity, the particle will experience the spatial and temporal variation of field. Assuming that the reference particle is synchronized with the cavity wave, the equation of motion in Eq. (30) can be rewritten by, for a particle with a longitudinal distance $z$ from the synchronous particle,

$$
\begin{aligned}
& \frac{d p_{x}}{d t}=\frac{q}{p_{0}} E_{x}(x, \beta c t) \cos (k y) \sin \left(\omega\left(t-\frac{z}{\beta c}\right)\right), \\
& \frac{d p_{y}}{d t}=-\frac{q}{p_{0}} \frac{\beta c}{Z_{0}} E_{\sigma}(x, \beta c t) \sin \left(\frac{2 \pi y}{\lambda}\right) \cos \left(\omega\left(t-\frac{z}{\beta c}\right)\right), \\
& \frac{d p_{z}}{d t}=\frac{q}{p_{0}} E_{\sigma}(x, \beta c t) \cos (k y) \sin \left(\omega\left(t-\frac{z}{\beta c}\right)\right) .
\end{aligned}
$$

The reference particle passes through the cavity gap in time $t \in n T_{0}+\left(-L_{\sigma} / 2 \beta c, L_{\sigma} / 2 \beta c\right)$, where $L_{\sigma}$ is the cavity gap width along the $\sigma$ direction. In Eq. (31), the temporal variable $t$ is synchronized with on-momentum particle in the center of the cavity, i.e., the reference particle sees zero field at $t=n T_{0}$ or simply $t=0$. Off-momentum particles see temporal variation of field different from the field variation which on-momentum particle sees. Hence, $t$ is replaced by $t-\frac{z}{\beta c}$, and $\sigma$ is by $\beta c t$. The cavity kick for a particle per passage is not available for analytical formula.

In a real application, the electric and magnetic fields are not given by a functional form, for an example, such as Eq. (29). The fields are given by numerical data at finite number of grid points. In order to simulate the real application, we generate the electromagnetic fields using Eq. (29). The spatial domain is $-0.1 \leq x \leq 0.1,-0.1 \leq y \leq 0.1$, and $-L_{\sigma} / 2 \leq z \leq L_{\sigma} / 2$. 


\begin{tabular}{|c|c|c|c|}
\hline Parameter & Unit & Rectangular shape & Wire model \\
\hline \hline Frequency of $\pi$ mode & $\mathrm{MHz}$ & 400 & 400 \\
\hline$\lambda / 2$ of $\pi$ mode & $\mathrm{mm}$ & 374.7 & 374.7 \\
\hline Frequency of 0 mode & $\mathrm{MHz}$ & 411.0 & - \\
\hline Nearest mode of $\pi$ mode & $\mathrm{MHz}$ & 411.0 & - \\
\hline Cavity reference length & $\mathrm{mm}$ & 444.7 & 444.7 \\
\hline Cavity width & $\mathrm{mm}$ & 300.0 & 300.0 \\
\hline Cavity height & $\mathrm{mm}$ & 383.2 & 383.2 \\
\hline Bars length & $\mathrm{mm}$ & 330.0 & - \\
\hline Bars width & $\mathrm{mm}$ & 55.0 & - \\
\hline Aperture diameter & $\mathrm{mm}$ & 84.0 & 84.0 \\
\hline Deflecting voltage $\left(V_{T}\right)$ & $\mathrm{MV}$ & 0.375 & - \\
\hline Peak electric field $\left(E_{P}\right)$ & $\mathrm{MV} / \mathrm{m}$ & 2.2 & - \\
\hline Peak magnetic field $\left(B_{P}\right)$ & $\mathrm{mT}$ & 7.9 & - \\
\hline$B E_{P} / E_{P}$ & $\mathrm{mT} /(\mathrm{MV} / \mathrm{m})$ & 3.6 & - \\
\hline Geometrical factor $\left(G=Q R_{s}\right)$ & $\Omega$ & 74.1 & - \\
\hline$[R / Q]_{T}$ & $\Omega$ & 413.34 & \\
\hline
\end{tabular}

Table I: Cavity parameters. The parameters of rectangular shape is taken from [2].

The number of grid points is $\left(N_{x}, N_{y}, N_{z}\right)=(20,20,40)$. Table I summaries the parameters of cavity. We use the cavity parameters: the cavity frequency $f=400 \mathrm{MHz}$, the cavity length $L_{\sigma}=0.4447 \mathrm{~m}$, the cavity width $W_{x}=300.0$, the cavity bar position $a=0.125 \mathrm{~m}$, the cavity height $\frac{\lambda}{2}=0.3832 \mathrm{~m}$.

3) Parallel bar model (Numerical field data from ODU): The electric and magnetic field of actual cavity design contains all vector components, i.e., $\mathbf{E}=E_{x} \hat{x}+E_{y} \hat{y}+E_{z} \hat{z}$ and $\mathbf{H}=H_{x} \hat{x}+H_{y} \hat{y}+H_{z} \hat{z}$. In general, the electric and magnetic fields are

$$
\begin{aligned}
& \mathbf{E}(x, y, z, t)=\mathbf{E}(x, y, \beta c t) \sin \left(\omega\left(t-\frac{z}{\beta c}\right)\right), \\
& \mathbf{H}(x, y, z, t)=\mathbf{H}(x, y, \beta c t) \cos \left(\omega\left(t-\frac{z}{\beta c}\right)\right) .
\end{aligned}
$$

Then the equation of motion becomes

$$
\begin{aligned}
& \frac{d p_{x}}{d t}=\frac{q}{p_{0}} E_{x}(x, y, \beta c t) \sin \left(\omega t\left(-\frac{z}{\beta c}\right)\right)-\frac{q \beta c}{p_{0}} \frac{1}{\mu_{0}} H_{y}(x, y, \beta c t) \cos \left(\omega\left(t-\frac{z}{\beta c}\right)\right), \\
& \frac{d p_{y}}{d t}=\frac{q}{p_{0}} E_{y}(x, y, \beta c t) \sin \left(\omega\left(t-\frac{z}{\beta c}\right)\right)+\frac{q \beta c}{p_{0}} \frac{1}{\mu_{0}} H_{x}(x, y, \beta c t) \cos \left(\omega\left(t-\frac{z}{\beta c}\right)\right), \\
& \frac{d p_{z}}{d t}=\frac{q}{p_{0}} E_{\sigma}(x, y, \beta c t) \sin \left(\omega\left(t-\frac{z}{\beta c}\right)\right) .
\end{aligned}
$$

Figure 7 shows the cavity kicks which are calculated using the electromagnetic field data of JLab design at $y=0$ plane. The cavity kicks are calculated in domain $-0.05 \leq x \leq 0.05,-0.05 \leq x \leq 0.05$, and $-0.23 \leq z \leq 0.23$ in units of meter because the aperture diameter of the cavity is $0.084 \mathrm{~m}$. The increment of grid points is $0.01 \mathrm{~m}$. The number of grid points is $\left(n_{x}, n_{y}, n_{z}\right)=(11,11,47)$. Parallel wire and parallel bar models show the same result (see Fig. 5 and Fig. 7).

Implementation in the bbsimc: The electric and magnetic field data obtained from the ODU should be rearranged in order to use them in the bbsimc code. First of all, the aperture which a beam particle passes through is much smaller than the cavity dimension. The field data inside the aperture is only required. Second, the field profile does not depend on a RF voltage. Therefore, the field data is extracted in domain $-0.05 \leq x \leq 0.05,-0.05 \leq x \leq 0.05$, and $-0.23 \leq z \leq 0.23$ in units of meter because the aperture diameter of the cavity is $0.084 \mathrm{~m}$. The cavity kicks are calculated in the domain. In order to determine the field strength, the transverse deflecting voltage is defined as

$$
V_{T, r m s}=\left(\int_{-L / 2}^{L / 2} d z|\mathbf{E}(0,0, z)|^{2}\right)^{1 / 2}
$$

The value of the cavity kicks is adjusted by

$$
\Delta p_{x, y, z}=\Delta p_{x, y, z} \times \frac{V_{c c}}{V_{T, r m s}}
$$

where $V_{c c}$ is the cavity voltage.

- Check symplecticity from the Jacobian matrix for different grid sizes. 


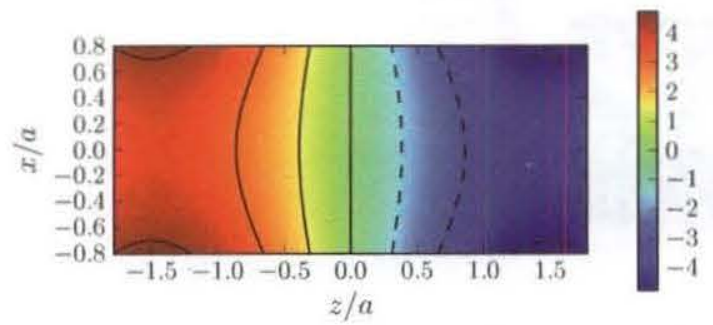

(a)

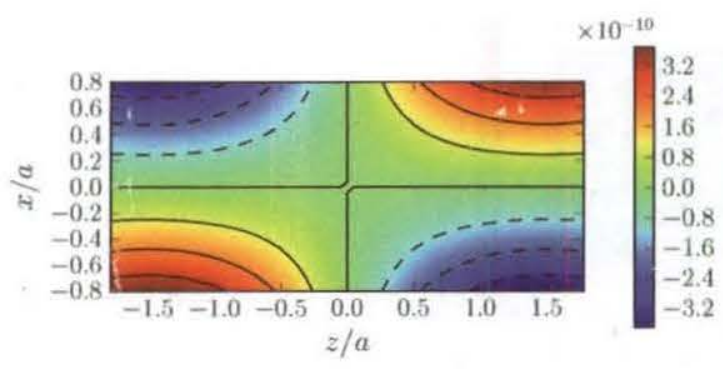

(c)

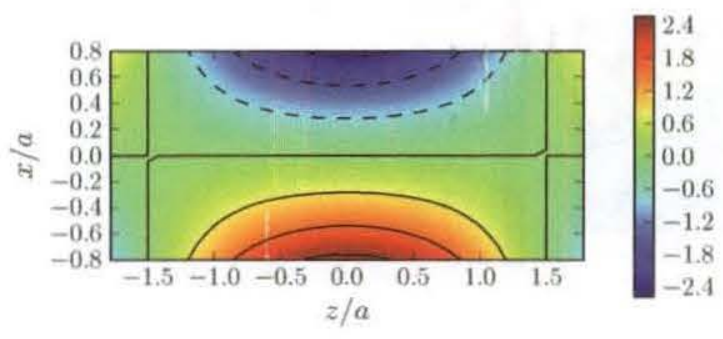

(e)

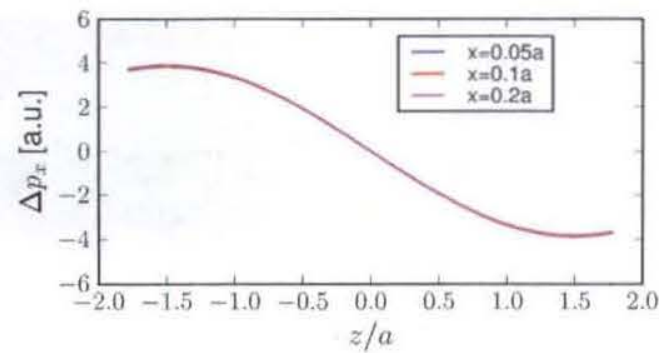

(b)

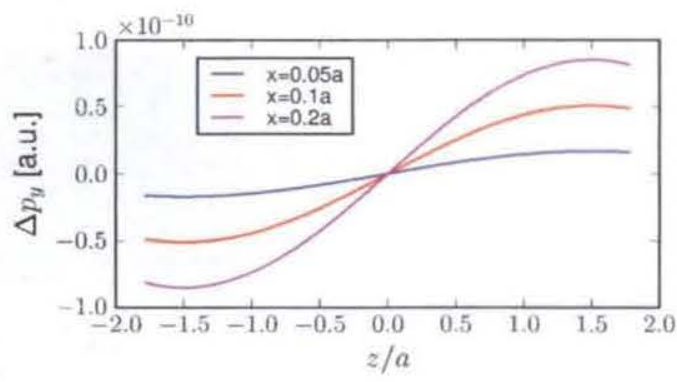

(d)

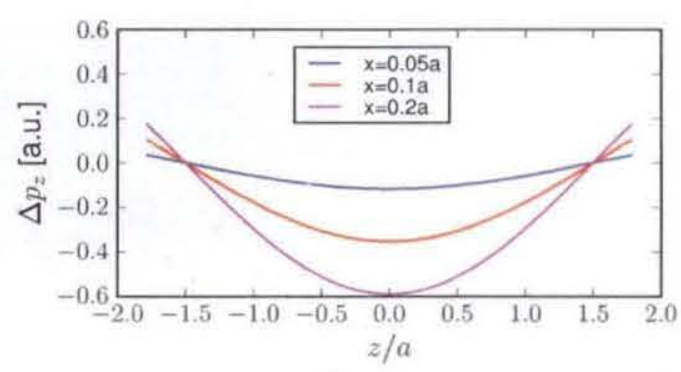

(f)

Figure 5: Plot of the cavity kicks which are calculated using the functional form of the electric and magnetic fields at $y=0$ plane: (a)-(b) horizontal kicks, (c)-(d) vertical kicks, and (e)-(f) longitudinal kicks. In this plot, $\beta=1, \omega=400 \mathrm{MHz}$, the cavity gap $L_{\sigma}=0.4447 \mathrm{~m}$, the bar position $a=0.125 \mathrm{~m}$, and the cavity height $\frac{\lambda}{2}=0.3832 \mathrm{~m}$ are applied.

\section{REFERENCES}

[1] J. Delayen and H. Wang, "New compact TEM-type deflecting and crabbing rf structure," Phys. Rev. Spec. Top. Acceler. Beams, vol. 12, p. $062002,2009$.

[2] J. Delayen, "Update on ODU/Л AB compact cavity design," May 2011. [Online]. Available: https://indico.fnal.gov/conferenceDisplay.py?confld=4041

[3] G. Ripken, "Nonlinear Canonical Equations Of Coupled Synchrobetatron Motion And Their Solution Within The Framework Of A Nonlinear SixDimensional (Symplectic) Tracking Program For Ultrarelativistic Protons," DESY, Tech. Rep. 85-084, 1985.

[4] G. Ripken and F. Schmidt, "A Symplectic Six-Dimensional Thin-Lens Formalism for Tracking," CERN, Tech. Rep. SL-95-12, 1995.

[5] W. Brittin, W. Smythe, and W. Wyss, "Poincaré gauge in electrodynamics," American Journal of Physics, vol. 50, p. 693, 1982.

\section{APPENDIX}

\section{A. Hamiltonian}

The Hamiltonian in the natural coordinates $(x, y, s)$ is given by [3]

$$
\mathcal{H}=\frac{m_{0} c^{2}}{\sqrt{1-v^{2} / c^{2}}}+q \phi .
$$

The Hamiltonian $\mathcal{H}$ is the sum of the mechanical and field energy. The Hamiltonian of charged particle in an electromagnetic field is given by

$$
\mathcal{H}\left(x, y, s, p_{x}, p_{y}, p_{s}, t\right)=c\left[m_{0}^{2} c^{2}+(\mathbf{p}-q \mathbf{A})^{2}\right]^{1 / 2}+q \phi
$$




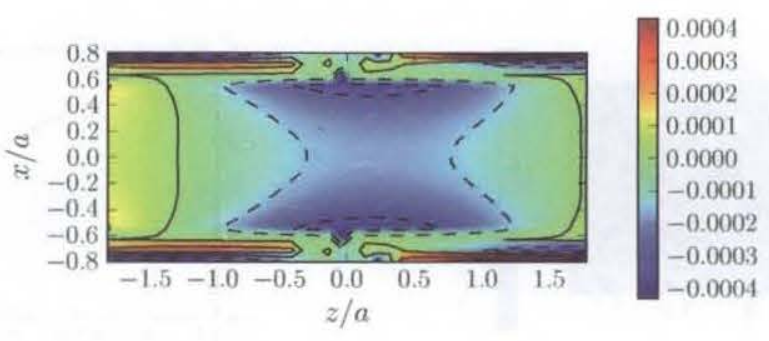

(a)

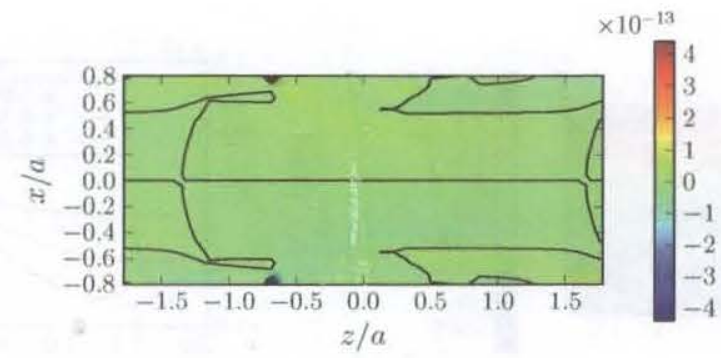

(b)

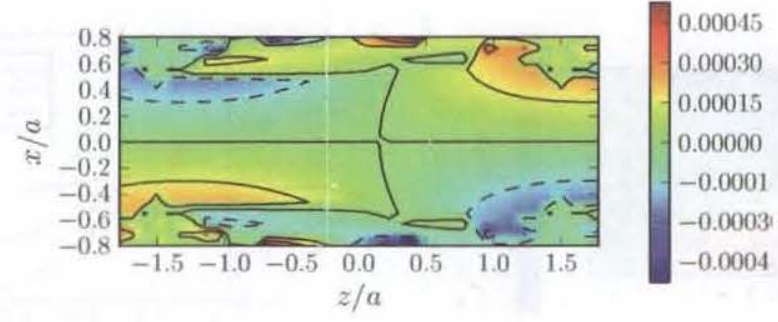

(c)

Figure 6: Plot of the difference of crab cavity kicks calculated by the functional fields and the numerical data at grid points at $y=0$ plane. In this plot, $\beta=1, \omega=400 \mathrm{MHz}$, the cavity gap $L_{\sigma}=0.4447 \mathrm{~m}$, the bar position $a=0.125 \mathrm{~m}$, and the cavity height $\frac{\lambda}{2}=0.3832 \mathrm{~m}$ are applied.

where the longitudinal momentum is $p_{s} /\left(1+h_{x} x+h_{y} y\right)$ for the curvatures $h_{x}$ and $h_{y}$ in the $x$-direction and in the $y$-direction respectively. Usually in accelerator physics, the arc length $s$ of the design orbit is introduced as independent variable. The new Hamiltonian is

$$
\begin{aligned}
\mathcal{K}\left(x, y, t, p_{x}, p_{y},-\mathcal{H}, s\right)= & -p_{s}, \\
= & -\left(1+h_{x} x+h_{y} y\right)\left[\frac{1}{c^{2}}(\mathcal{H}-e \phi)^{2}-m_{0}^{2} c^{2}-\left(p_{x}-q A_{x}\right)^{2}-\left(p_{y}-q A_{y}\right)^{2}\right]^{1 / 2} \\
& -\left(1+h_{x} x+h_{y} y\right) q A_{s} .
\end{aligned}
$$

Now, $t$ and $-\mathcal{H}$ form a pair of new conjugated canonical variable. The equation of motion is

$$
\begin{aligned}
\frac{d x}{d s} & =\frac{\partial \mathcal{K}}{\partial p_{x}}, \quad \frac{d y}{d s}=\frac{\partial \mathcal{K}}{\partial p_{y}} \quad \frac{d t}{d s}=\frac{\partial \mathcal{K}}{\partial(-\mathcal{H})}, \\
\frac{d p_{x}}{d s} & =-\frac{\partial \mathcal{K}}{\partial x}, \quad \frac{d p_{y}}{d s}=-\frac{\partial \mathcal{K}}{\partial y} \quad \frac{d(-\mathcal{H})}{d s}=-\frac{\partial \mathcal{K}}{\partial t} .
\end{aligned}
$$

In the following we choose a gauge with $\phi=0$ (e.g. Coulomb gauge). Instead of using $t$ and $\mathcal{H}$, we introduce new variables $(-c t, \eta)$,

$$
\eta=\frac{E-E_{0}}{E_{0}},
$$




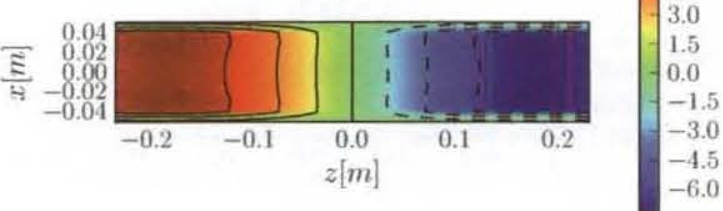

(a)

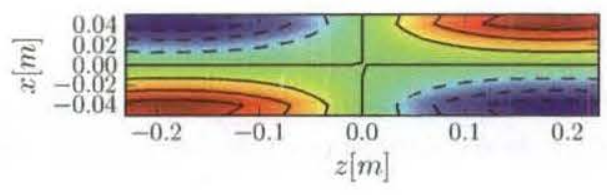

(c)

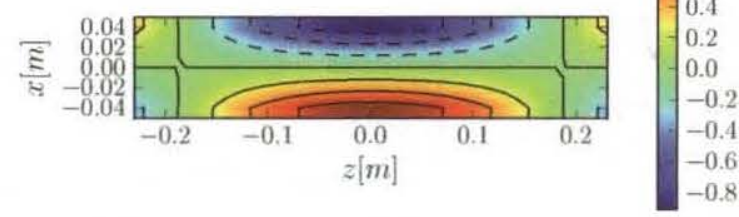

(e)

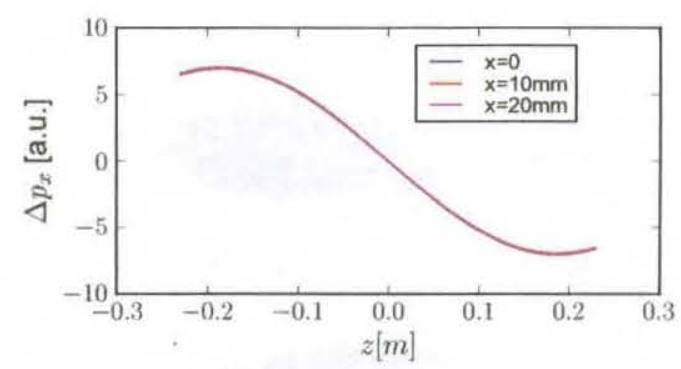

(b)

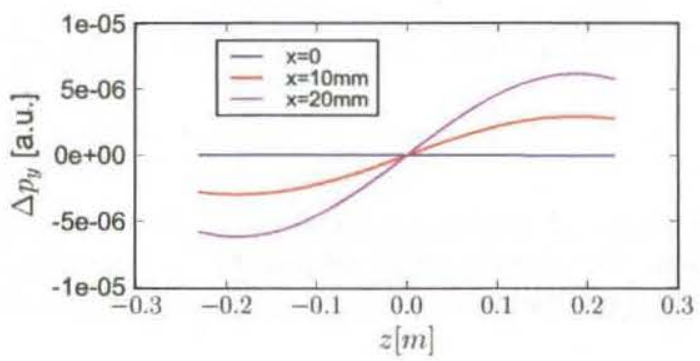

(d)

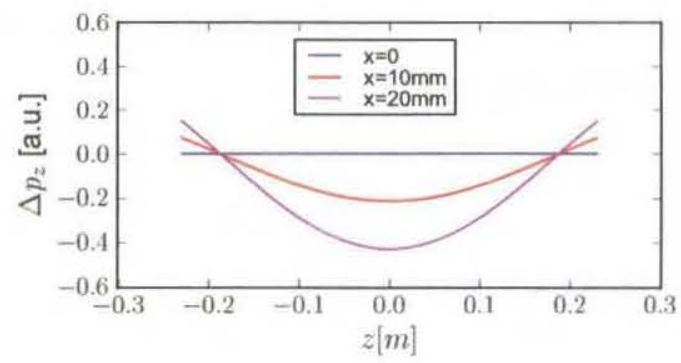

(f)

Figure 7: Plot of the cavity kicks which are calculated using the electromagnetic field data of $J$ Lab design at $y=0$ plane: (a)-(b) horizontal kicks, (c)-(d) vertical kicks, and (e)-(f) longitudinal kicks. In this plot, $\beta=1, \omega=400 \mathrm{MHz}$, the cavity gap $L_{\sigma}=0.4447 \mathrm{~m}$, and the cavity height $\frac{\lambda}{2}=0.3832 \mathrm{~m}$ are applied.

where the particle energy $E \equiv \mathcal{H}$, and the design energy $E_{0}$. The Hamiltonian becomes

$$
\begin{aligned}
\overline{\mathcal{K}}\left(x, y,-c t, p_{x}, p_{y}, \eta, s\right)= & \frac{1}{p_{0}} \mathcal{K}, \\
= & -\left(1+h_{x} x+h_{y} y\right)\left[(1+\hat{\eta})-\left(\bar{p}_{x}-\frac{q}{p_{0}} A_{x}\right)^{2}-\left(\bar{p}_{y}-\frac{q}{p_{0}} A_{y}\right)^{2}\right]^{1 / 2} \\
& -\left(1+h_{x} x+h_{y} y\right) \frac{q}{p_{0}} A_{s} .
\end{aligned}
$$

Here $p_{0}$ and $E_{0}$ are the design momentum and energy, $p_{0}=E_{0} \beta_{0} / c$. In general, $E=m_{0} \gamma c^{2}, p=m_{0} \gamma v, \gamma=m_{0} c^{2} / E=$ $1 / \sqrt{1-\beta^{2}}$, and $\beta=\sqrt{1-\left(m_{0} c^{2} / E\right)^{2}}$. The new variable $\hat{\eta}$ is

$$
\hat{\eta}=\frac{1}{\beta_{0}} \sqrt{(1+\eta)^{2}-\left(\frac{m_{0} c^{2}}{E_{0}}\right)^{2}} .
$$

Since the variable $t$ increases without limit, it is more useful to introduce the variable $z=s-c t$ which describes the delay in arrival time at position $s$ of a particle traveling at the speed of light $c$. The canonical transformation can be achieved using 


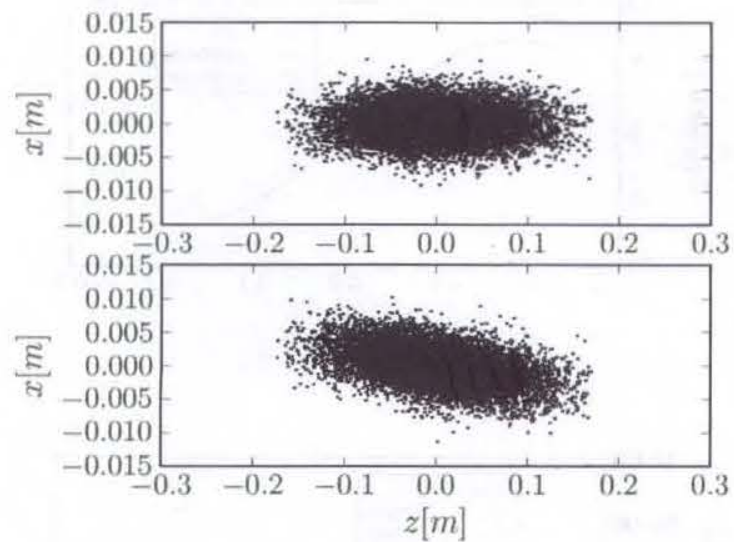

(a)

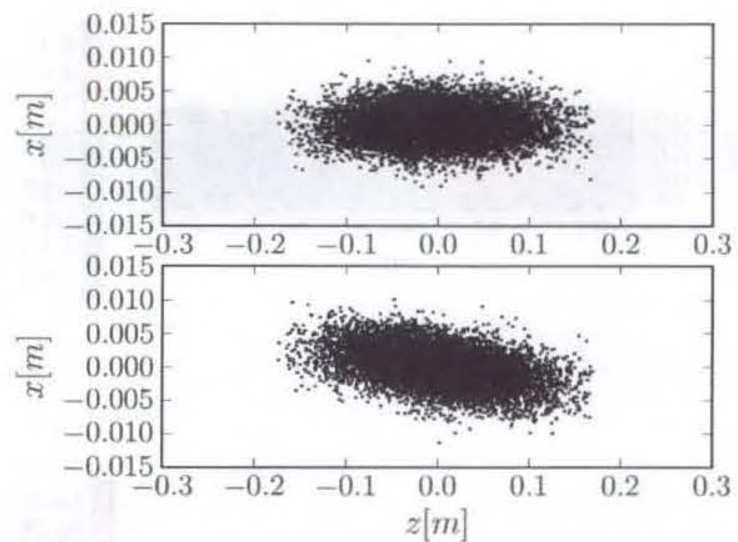

(b)

Figure 8: Plot of deflecting angle due to (a) TM-mode crab cavity and (b) TEM-mode crab cavity. Top plots show the initial distribution. Bottom plots show the distribution after crab cavity kicks. To illustrate the deflecting angle, large cavity voltage is applied. The same crab voltage is applied to TM and TEM cavities.

the generating function

$$
F_{3}\left(\bar{x}, \bar{y}, z, p_{x}, p_{y}, \eta, s\right)=-p_{x} \bar{x}-p_{y} \bar{y}-z \eta+s \eta
$$

which gives transformations:

$$
\begin{gathered}
x=-\frac{\partial F_{3}}{\partial p_{x}}=\bar{x}, \quad y=\bar{y}, \quad-c t=z-s, \\
\bar{p}_{x}=-\frac{\partial F_{3}}{\partial \bar{x}}=p_{x}, \quad \bar{p}_{y}=p_{y}, \quad \bar{\eta}=\eta .
\end{gathered}
$$

Finally we get the Hamiltonian [4]

$$
\begin{aligned}
\mathcal{H}\left(x, p_{x}, y, p_{y}, z, p_{z} ; s\right)= & -\left(1+f\left(p_{z}\right)\right)\left(1+h_{x} x+k_{y} y\right)\left(1-\frac{\left(p_{x}-\frac{q}{p_{0}} A_{x}\right)^{2}+\left(p_{y}-\frac{q}{p_{0}} A_{y}\right)^{2}}{\left(1+f\left(p_{z}\right)\right)^{2}}\right)^{1 / 2} \\
& -\left(1+h_{x} x+h_{y} y\right) \frac{q}{p_{0}} A_{s} .
\end{aligned}
$$

Here we define $f\left(p_{z}\right) \equiv \hat{\eta}$ and $p_{z}=\eta / \beta_{0}^{2} . h_{x}$ and $h_{y}$ denote the curvatures in the $x$-direction and in the $y$-direction respectively. $A_{x}, A_{y}$ and $A_{s}$ denote the vector potentials. The longitudinal coordinates $\left(z, p_{z}\right)$ are defined as $\left(s-v_{0} t, \frac{1}{\beta_{0}^{2}} \eta\right)$. The variable $z$ describes the delay in arrival time at position $s$ of a particle and is the longitudinal separation of the particle from the center of the bunch. The quantity $\eta$ is the relative energy deviation of the particle defined by $\eta=\Delta E / E_{0}$. $E_{0}$ is the design energy. The relative momentum derivation $\hat{\eta} \equiv f\left(p_{z}\right)$ is defined as

$$
\begin{aligned}
\hat{\eta} & =\frac{p}{p_{0}}-1=\frac{\Delta p}{p_{0}}, \\
& =\frac{1}{\beta_{0}} \sqrt{(1+\eta)^{2}-\left(\frac{m_{0} c^{2}}{E_{0}}\right)^{2}}-1, \\
& =\frac{1}{\beta_{0}} \sqrt{\left(1+\beta_{0}^{2} p_{z}\right)^{2}-\left(\frac{m_{0} c^{2}}{E_{0}}\right)^{2}}-1, \\
& =\sqrt{1+2 p_{z}+\beta_{0}^{2} p_{z}^{2}}-1 .
\end{aligned}
$$


Note that $\gamma \equiv \frac{E}{m_{0} c^{2}}=1 / \sqrt{1-\beta^{2}}, \beta=\sqrt{1-\gamma^{-2}}, E=m_{0} \gamma c^{2}, p=m_{0} \gamma v$, and design velocity $v_{0}=c \beta_{0} .1+\hat{\eta}=\frac{p}{p_{0}}$. The corresponding canonical equations read as

$$
\begin{aligned}
\frac{d}{d s} x & =+\frac{\partial \mathcal{H}}{\partial p_{x}}, & \frac{d}{d s} p_{x} & =-\frac{\partial \mathcal{H}}{\partial x}, \\
\frac{d}{d s} y & =+\frac{\partial \mathcal{H}}{\partial p_{y}}, & \frac{d}{d s} p_{y} & =-\frac{\partial \mathcal{H}}{\partial y}, \\
\frac{d}{d s} z & =+\frac{\partial \mathcal{H}}{\partial p_{z}}, & \frac{d}{d s} p_{z} & =-\frac{\partial \mathcal{H}}{\partial z} .
\end{aligned}
$$

In order to utilize this Hamiltonian, the electric field $\vec{E}$ and the magnetic field $\vec{B}$ or the corresponding vector potential $\vec{A}(x, y, z ; s)$ for the accelerator elements must be given. Once $\vec{A}$ is known, the fields $\vec{E}$ and $\vec{B}$ may be found

$$
\vec{E}=-\frac{\partial \mathbf{A}}{\partial t}=\beta_{0} c \frac{\partial}{\partial z} \vec{A}
$$

and $\mathbf{B}=\nabla \times \mathbf{A}$,

$$
\begin{aligned}
& B_{x}=\frac{1}{1+h_{x} x+h_{y} y}\left\{\frac{\partial}{\partial y}\left[\left(1+h_{x} x+h_{y} y\right) A_{s}\right]-\frac{\partial}{\partial s} A_{y}\right\}, \\
& B_{y}=\frac{1}{1+h_{x} x+h_{y} y}\left\{\frac{\partial}{\partial s} A_{x}-\frac{\partial}{\partial x}\left[\left(1+h_{x} x+h_{y} y\right) A_{s}\right]\right\}, \\
& B_{z}=\frac{\partial}{\partial x} A_{y}-\frac{\partial}{\partial y} A_{x} .
\end{aligned}
$$

Since the transverse momenta are much smaller than the total momentum $p$, i.e., $\left|p_{x}-\frac{q}{p_{0} c} A_{x}\right| \ll \frac{p}{p_{0}}$ and $\left|p_{y}-\frac{q}{p_{0} c} A_{y}\right| \ll \frac{p}{p_{0}}$, one can expand the Hamiltonian up to second order in $p_{x}, p_{y}$ :

$$
\begin{aligned}
\mathcal{H}= & p_{z}-\left(1+f\left(p_{z}\right)\right)\left(1+h_{x} x+k_{y} y\right)\left(1-\frac{1}{2} \frac{\left(p_{x}-\frac{q}{p_{0}} A_{x}\right)^{2}+\left(p_{y}-\frac{q}{p_{0}} A_{y}\right)^{2}}{\left(1+f\left(p_{z}\right)\right)^{2}}+\ldots\right) \\
& -\left(1+h_{x} x+h_{y} y\right) \frac{q}{p_{0}} A_{s}, \\
\simeq & \left(1+h_{x} x+k_{y} y\right) \frac{\left(p_{x}-\frac{q}{p_{0}} A_{x}\right)^{2}+\left(p_{y}-\frac{q}{p_{0}} A_{y}\right)^{2}}{2\left(1+f\left(p_{z}\right)\right)} \\
& +p_{z}-\left(1+h_{x} x+h_{y} y\right)\left(1+f\left(p_{z}\right)+\frac{q}{p_{0}} A_{s}\right) .
\end{aligned}
$$

For an example, the vector potential $\vec{A}$ of common accelerator elements such as bending magnets, quadrupoles, skew quadrupoles, sextupoles, octupoles, solenoids and cavities can be written as:

$$
\begin{aligned}
\frac{q}{p_{0} c} A_{s}= & -\frac{1}{2}\left(1+h_{x} x+h_{y} y\right)+\frac{1}{2} g\left(y^{2}-x^{2}\right)+N x y-\frac{1}{6}\left(x^{3}-3 x y^{2}\right)+\frac{\mu}{24}\left(y^{4}-6 x^{2} y^{2}+x^{4}\right) \\
& -\frac{1}{\beta_{0}^{2}} \frac{L}{2 \pi h} \frac{q V(s)}{E_{0}} \cos \left[h \frac{2 \pi}{L} z+\varphi\right], \\
\frac{q}{p_{0} c} A_{x}= & -H y, \\
\frac{q}{p_{0} c} A_{y}= & +H x,
\end{aligned}
$$

where $h$ is the harmonic number. Above abbreviations represent:

1) bending magnet: $h_{x}^{2}+h_{y}^{2} \neq 0$.

2) quadrupole: $g=\frac{q}{p_{0} c}\left(\frac{\partial B_{y}}{\partial x}\right)_{x=y=0}$.

3) skew quadrupole: $N=\frac{1}{2} \frac{q}{p_{0} c}\left(\frac{\partial B_{x}}{\partial x}-\frac{\partial B_{y}}{\partial y}\right)_{x=y=0}$.

4) sextupole: $\lambda=\frac{q}{p_{0} c}\left(\frac{\partial^{2} B_{y}}{\partial x^{2}}\right)_{x=y=0}$.

5) octupole: $\mu=\frac{q}{p_{0} c}\left(\frac{\partial^{3} B_{y}}{\partial x^{3}}\right)_{x=y=0}$.

6) solenoid: $H=\frac{1}{2} \frac{q}{p_{0} c} B_{s}(0,0, s)$.

7) main rf cavity: $V \neq 0$. 


\section{B. Lorentz force}

Let us derive the Lorentz force equation from the Hamiltonian. The canonical momentum is defined by

$$
\mathbf{p}=m \mathbf{v}+\frac{q}{p_{0}} \mathbf{A}=m \frac{d \mathbf{x}}{d t}+\frac{q}{p_{0}} \mathbf{A} .
$$

The time derivative of canonical momentum gives

$$
\frac{d p_{i}}{d t}=m \frac{d^{2} x_{i}}{d t^{2}}+\frac{q}{p_{0}} \frac{d}{d t} A_{i}=\frac{d^{2} x_{i}}{d t^{2}}+\frac{q}{p_{0}}\left(\frac{\partial A_{i}}{\partial t}+(\mathbf{v} \cdot \nabla) \mathbf{A}\right),
$$

where the subscript $i$ represents the coordinates $i=x, y, z$. From the Hamilton equation,

$$
\begin{aligned}
\frac{d p_{i}}{d s} & =-\frac{\partial H}{\partial x_{i}} \\
& =\frac{\left(\mathbf{p}-\frac{q}{p_{0}} \mathbf{A}\right)}{1+f\left(p_{z}\right)} \cdot \frac{q}{p_{0}} \frac{\partial \mathbf{A}}{\partial x_{i}}-\frac{\left(p_{z}-\frac{q}{p_{0}} A_{z}\right)}{1+f\left(p_{z}\right)} \frac{q}{p_{0}} \frac{\partial A_{z}}{\partial x_{i}}+\frac{q}{p_{0}} \frac{\partial A_{z}}{\partial x_{i}} .
\end{aligned}
$$

Using $z=s-v_{0} t$ and $d s=v_{0} d t$, Eq. (53) and (54) gives

$$
\frac{d^{2} x_{i}}{d t^{2}}=v_{0} \frac{d p_{i}}{d s}-\frac{q}{p_{0}}\left(\frac{\partial A_{i}}{\partial t}+(\mathbf{v} \cdot \nabla) \mathbf{A}_{i}\right) .
$$

Note that $p_{0}=m v_{0}$. Since $\mathbf{p}-\frac{q}{p_{0}} \mathbf{A}=\frac{m \mathbf{v}}{p_{0}}, v_{0}\left(\mathbf{p}-\frac{q}{p_{0}} \mathbf{A}\right)$ becomes $\mathbf{v}$. Then, above equation becomes

$$
\begin{aligned}
m \frac{d^{2} x_{i}}{d t^{2}} & =\frac{\mathbf{v}}{1+f\left(p_{z}\right)} \cdot \frac{q}{p_{0}} \frac{\partial \mathbf{A}}{\partial x_{i}}-\frac{v_{z}}{1+f\left(p_{z}\right)} \frac{q}{p_{0}} \frac{\partial A_{z}}{\partial x_{i}}+\frac{q v_{0}}{p_{0}} \frac{\partial A_{z}}{\partial x_{i}}-\frac{q}{p_{0}}\left(\frac{\partial A_{i}}{\partial t}+(\mathbf{v} \cdot \nabla) \mathbf{A}_{i}\right), \\
& =\frac{q}{p_{0}} E_{i}+\frac{q}{p_{0}}\left[\frac{\mathbf{v}}{1+f\left(p_{z}\right)} \cdot \frac{\partial \mathbf{A}}{\partial x_{i}}-(\mathbf{v} \cdot \nabla) \mathbf{A}_{i}+\left(v_{0}-\frac{v_{z}}{1+f\left(p_{z}\right)}\right) \frac{\partial A_{z}}{\partial x_{i}}\right], \\
& =\frac{q}{p_{0}} E_{i}+\frac{q}{p_{0}}\left[\mathbf{v} \cdot \frac{\partial \mathbf{A}}{\partial x_{i}}-(\mathbf{v} \cdot \nabla) \mathbf{A}_{i}-\frac{f\left(p_{z}\right)}{1+f\left(p_{z}\right)} \mathbf{v} \cdot \frac{\partial \mathbf{A}}{\partial x_{i}}+\left(v_{0}-\frac{v_{z}}{1+f\left(p_{z}\right)}\right) \frac{\partial A_{z}}{\partial x_{i}}\right], \\
& =\frac{q}{p_{0}}\left[E_{i}+(\mathbf{v} \times \mathbf{B})_{i}\right]+\frac{q}{p_{0}}\left[-\frac{f\left(p_{z}\right)}{1+f\left(p_{z}\right)} \mathbf{v} \cdot \frac{\partial \mathbf{A}}{\partial x_{i}}+\left(v_{0}-\frac{v_{z}}{1+f\left(p_{z}\right)}\right) \frac{\partial A_{z}}{\partial x_{i}}\right],
\end{aligned}
$$

where $E_{i}=-\frac{q}{p_{0}} \frac{\partial A_{i}}{\partial t}$ and $(\mathbf{v} \times \mathbf{B})_{i}=\mathbf{v} \cdot \frac{\partial \mathbf{A}}{\partial x_{i}}-(\mathbf{v} \cdot \nabla) \mathbf{A}_{i}$. Now, consider special two cases: (1) TM mode cavity $\left(\mathbf{v}=v_{0} \hat{z}\right.$ and $\left.\mathbf{A}=A_{z} \hat{z}\right)$, and (2) TEM mode cavity $\left(\mathbf{v}=v_{0} \hat{z}\right.$ and $\left.\mathbf{A}=A_{x} \hat{x}+A_{z} \hat{z}\right)$. Since $\mathbf{v} \cdot \frac{\partial \mathbf{A}}{\partial x_{i}}=v_{0} \frac{\partial A_{z}}{\partial x_{i}}$ and $v_{z}=v_{0}$ for both cases, Eq. (56) becomes

$$
\frac{d}{d t} \mathbf{P}=\frac{q}{p_{0}}(\mathbf{E}+\mathbf{v} \times \mathbf{B})
$$

where $\mathbf{P}$ is the mechanical momentum normalized by $p_{0}$. (Think: Above result is only applicable for a design orbit particle. For a design orbit, $v_{x}=v_{y}=0$ and $\mathbf{v}=p_{0} / m \hat{z}=v_{0} \hat{z} \cdot p_{0}=\left(p_{x}^{2}+p_{y}^{2}+p_{z}^{2}\right)^{1 / 2}$. However, in general, $p_{x} \neq 0, p_{y} \neq 0$, and $p_{z} \neq 0$.).

C. TM mode cavity - Hamiltonian

D. Horizontal crab cavity

Since there is only longitudinal electric field in the TM110, one can get the vector potential of TM110 mode using Eq. (48) and (4)

$$
A_{s}(x, y, \sigma, t)=\frac{1}{\omega} E_{0} J_{1}\left(\frac{\alpha}{R} r\right) \frac{x}{r} \sin \left(\omega t-\frac{\omega}{\beta c} z\right) .
$$

Since the Hamiltonian is described by the longitudinal coordinate $s$ instead of time $t$, the vector potential should be expressed by, in terms of $s=\beta c t$,

$$
A_{s}(x, y, \sigma, s)=\frac{1}{\omega} E_{0} J_{1}\left(\frac{\alpha}{R} r\right) \frac{x}{r} \sin \left(\frac{\omega}{\beta c}(s-z)\right) .
$$


Note that $z$ is the longitudinal distance from the synchronous particle. As above, the reference particle is synchronized with the cavity. Equation (47) gives the equation of motion:

$$
\begin{aligned}
\frac{d}{d s} x & =\frac{p_{x}}{1+f\left(p_{z}\right)}, \\
\frac{d}{d s} p_{x} & =-\frac{q}{p_{0} c} E_{0}\left[\left(\frac{J_{1}\left(\frac{\alpha}{R} r\right)}{\frac{\alpha}{R} r}-J_{2}\left(\frac{\alpha}{R} r\right)\right) \frac{x^{2}}{r^{2}}+\frac{J_{1}\left(\frac{\alpha}{R} r\right)}{\frac{\alpha}{R} r} \frac{y^{2}}{r^{2}}\right] \sin \left(\frac{\omega}{\beta c}(s-z)\right), \\
\frac{d}{d s} y & =\frac{p_{y}}{1+f\left(p_{z}\right)}, \\
\frac{d}{d s} p_{y} & =-\frac{q}{p_{0} c} E_{0}\left[\left(\frac{J_{1}\left(\frac{\alpha}{R} r\right)}{\frac{\alpha}{R} r}-J_{2}\left(\frac{\alpha}{R} r\right)\right)-\frac{J_{1}\left(\frac{\alpha}{R} r\right)}{\frac{\alpha}{R} r}\right] \frac{x y}{r^{2}} \sin \left(\frac{\omega}{\beta c}(s-z)\right), \\
\frac{d}{d s} z & =-\left(1+\frac{p_{x}^{2}+p_{y}^{2}}{2\left(1+f\left(p_{z}\right)\right)^{2}}\right) f^{\prime}\left(p_{z}\right), \\
\frac{d}{d s} p_{z} & =\frac{q}{p_{0} \beta c} E_{0} J_{1}\left(\frac{\alpha}{R} r\right) \frac{x}{r} \cos \left(\frac{\omega}{\beta c}(s-z)\right) .
\end{aligned}
$$

Here $\alpha / R=\omega / c$ is applied. The reference particle passes through the cavity gap, i.e., $s \in s_{0}+(-\lambda / 2, \lambda / 2)$, where $\lambda$ is the cavity gap width. The cavity kick for a particle per passage is

$$
\begin{aligned}
\Delta x & =\Delta y=\Delta z=0, \\
\Delta p_{x} & =\frac{q V_{c c}}{E}\left[\left(\frac{J_{1}\left(\frac{\alpha}{R} r\right)}{\frac{\alpha}{R} r}-J_{2}\left(\frac{\alpha}{R} r\right)\right) \frac{x^{2}}{r^{2}}+\frac{J_{1}\left(\frac{\alpha}{R} r\right)}{\frac{\alpha}{R} r} \frac{y^{2}}{r^{2}}\right] \sin \frac{\omega z}{\beta c}, \\
\Delta p_{y} & =\frac{q V_{c c}}{E}\left[\left(\frac{J_{1}\left(\frac{\alpha}{R} r\right)}{\frac{\alpha}{R} r}-J_{2}\left(\frac{\alpha}{R} r\right)\right)-\frac{J_{1}\left(\frac{\alpha}{R} r\right)}{\frac{\alpha}{R} r}\right] \frac{x y}{r^{2}} \sin \frac{\omega z}{\beta c}, \\
\Delta p_{z} & =\frac{q V_{c c}}{E} \frac{J_{1}\left(\frac{\alpha}{R} r\right)}{\frac{\alpha}{R} r} \frac{\omega x}{\beta c} \cos \frac{\omega z}{\beta c} .,
\end{aligned}
$$

where $q V_{c c}=q E_{0} \lambda T$ is the crab cavity voltage, $T=\sin \left(\frac{\omega \lambda}{2 \beta c}\right) /\left(\frac{\omega \lambda}{2 \beta c}\right)$ the transit time factor, and $E=p_{0} c$ the beam energy. Equation (61) is the same as Eq. (8).

\section{E. Vertical crab cavity}

For a vertical crab cavity, one can get the vector potential of TM110 mode by replacing $\phi$ as $\phi+\frac{\pi}{2}$ into the longitudinal electric field in Eq. (4)

$$
A_{s}(x, y, \sigma, s)=\frac{1}{\omega} E_{0} J_{1}\left(\alpha \frac{r}{R}\right) \frac{y}{r} \sin \left(\frac{\omega}{\beta c}(s-z)\right) .
$$

Note that $z$ is the longitudinal distance from the synchronous particle. Equation (47) gives the equation of motion:

$$
\begin{aligned}
\frac{d}{d s} x & =\frac{d}{d s} y=\frac{d}{d s} z=0, \\
\frac{d}{d s} p_{x} & =-\frac{q}{p_{0} c} E_{0}\left[-J_{2}\left(\alpha \frac{r}{R}\right)\right] \frac{x y}{r^{2}} \sin \left(\frac{\omega}{\beta c}(s-z)\right), \\
\frac{d}{d s} p_{y} & =-\frac{q}{p_{0} c} E_{0}\left[\frac{J_{1}\left(\alpha \frac{r}{R}\right)}{\alpha \frac{r}{R}}-J_{2}\left(\alpha \frac{r}{R}\right) \frac{y^{2}}{r^{2}}\right] \sin \left(\frac{\omega}{\beta c}(s-z)\right), \\
\frac{d}{d s} p_{z} & =\frac{q}{p_{0} \beta c} E_{0} J_{1}\left(\alpha \frac{r}{R}\right) \frac{y}{r} \cos \left(\frac{\omega}{\beta c}(s-z)\right) .
\end{aligned}
$$

The reference particle passes through the cavity gap, i.e., $s \in s_{0}+(-\lambda / 2, \lambda / 2)$, where $\lambda$ is the cavity gap width. The cavity kick for a particle per passage is

$$
\begin{aligned}
& \Delta p_{x}=\frac{q V_{c c}}{E}\left[-\left(\alpha \frac{r}{R}\right)^{-2} J_{2}\left(\alpha \frac{r}{R}\right)\right]\left(\alpha \frac{x}{R}\right)\left(\alpha \frac{y}{R}\right) \sin \frac{\omega z}{\beta c}, \\
& \Delta p_{y}=\frac{q V_{c c}}{E}\left[\left(\alpha \frac{r}{R}\right)^{-1} J_{1}\left(\alpha \frac{r}{R}\right)-\left(\alpha \frac{r}{R}\right)^{-2} J_{2}\left(\alpha \frac{r}{R}\right)\left(\alpha \frac{y}{R}\right)^{2}\right] \sin \frac{\omega z}{\beta c}, \\
& \Delta p_{z}=\frac{q V_{c c}}{E}\left(\alpha \frac{r}{R}\right)^{-1} J_{1}\left(\alpha \frac{r}{R}\right) \frac{\omega y}{\beta c} \cos \frac{\omega z}{\beta c},
\end{aligned}
$$


where $q V_{c c}=q E_{0} \lambda T$ is the crab cavity voltage, $T=\sin \left(\frac{\omega \lambda}{2 \beta c}\right) /\left(\frac{\omega \lambda}{2 \beta c}\right)$ the transit time factor, and $E=p_{0} c$ the beam energy. In the small beam size, i.e., $\frac{\alpha}{R} r \ll 1$, Eq. (64) can be approximate

$$
\begin{aligned}
& \Delta p_{x}=\frac{q \tilde{V}_{c c}}{E}\left[-\frac{1}{4}\left(\frac{\alpha}{R}\right)^{2}+\frac{1}{24}\left(\frac{\alpha}{R}\right)^{4} x^{2}+\cdots\right] x y \sin \frac{\omega z}{\beta c}, \\
& \Delta p_{y}=\frac{q \tilde{V}_{c c}}{E}\left[1-\frac{1}{8}\left(\frac{\alpha}{R}\right)^{2}\left(x^{2}-y^{2}\right)+\frac{1}{96}\left(\frac{\alpha}{R}\right)^{4}\left(x^{4}-y^{4}\right)+\cdots\right] \sin \frac{\omega z}{\beta c}, \\
& \Delta p_{s}=\frac{q \tilde{V}_{c c}}{E}\left[1-\frac{1}{8}\left(\frac{\alpha}{R}\right)^{2}\left(x^{2}-\frac{1}{3} y^{2}\right)+\frac{1}{96}\left(\frac{\alpha}{R}\right)^{4}\left(x^{4}-\frac{1}{5} y^{4}\right)+\cdots\right] \frac{\omega y}{\beta c} \cos \frac{\omega z}{\beta c} .
\end{aligned}
$$

In order to make the approximate solution be symplectic, Eq. (65) is rearranged in higher order terms. Note that the radius of KEK crab cavity is $R=0.433 \mathrm{~m}$ and $\alpha=3.832$. At the (proposed) crab cavity location of SPS, the rms beam size is $\sigma_{x}=0.91 \mathrm{~mm}$.

\section{F. Scalar and vector potential}

The homogeneous Maxwell equations

$$
\nabla \cdot \mathbf{B}=0
$$

and

$$
\nabla \times \mathbf{E}+\frac{\partial \mathbf{B}}{\partial t}=0
$$

imply the existence of scalar and vector potentials through which $\mathbf{E}$ and $\mathbf{B}$ may be expressed as [5]

$$
\mathbf{E}=-\nabla \phi-\frac{\partial \mathbf{A}}{\partial t}
$$

and

$$
\mathbf{B}=\nabla \times \mathbf{A} .
$$

That is, if $\mathbf{E}$ and $\mathbf{B}$ satisfy Eq. (66) and Eq. (67) then we may find $\phi$ and $\mathbf{A}$ such that Eq. (68) and Eq. (69) are valid. Conversely $\phi$ and $\mathbf{A}$ may be expressed in terms of the corresponding $\mathbf{E}$ and $\mathbf{B}$. The expressions for $\phi$ and $\mathbf{A}$ are not unique, however, because the gauge-transformed potential $\phi^{\prime}$ and $\mathbf{A}^{\prime}$ are

$$
\begin{aligned}
\phi^{\prime} & =\phi+\frac{\partial \chi}{\partial t}, \\
\mathbf{A}^{\prime} & =\mathbf{A}-\nabla \chi,
\end{aligned}
$$

where $\chi$ is an arbitrary smooth scalar, given the same $\mathbf{E}$ and $\mathbf{B}$.

Consider the averaged magnetic field for TEM mode cavity of parallel plate (see Eq. (15)):

$$
\begin{aligned}
\mathbf{B} & =\hat{\sigma} \frac{1}{c} \mathcal{E}_{0} \sin \left(\frac{2 \pi y}{\lambda}\right) \int_{-L / 2 \beta c}^{L / 2 \beta c} d t \cos \left(\omega\left(t-\frac{z}{\beta c}\right)\right), \\
& =\hat{\sigma} \frac{1}{c} \mathcal{E}_{0} \frac{2}{\omega} \sin \left(\frac{\omega L}{2 \beta c}\right) \sin \left(\frac{2 \pi y}{\lambda}\right) \cos \left(\frac{\omega z}{\beta c}\right), \\
& =\hat{\sigma} \frac{L}{\beta c^{2}} \mathcal{E}_{0} T \sin \left(\frac{2 \pi y}{\lambda}\right) \cos \left(\frac{\omega z}{\beta c}\right) .
\end{aligned}
$$

Equation (71) does not satisfy with $\nabla \cdot \mathbf{B}=0$.

\section{G. Useful formula}

The unit vector in cylindrical coordinates $(\hat{r}, \hat{\phi}, \hat{s})$ is related to the Cartesian basis $(\hat{x}, \hat{y}, \hat{s})$ :

$$
\begin{aligned}
\hat{r} & =\cos \phi \hat{x}+\sin \phi \hat{y}, \\
\hat{\phi} & =-\sin \phi \hat{x}+\cos \phi \hat{y}, \\
\hat{s} & =\hat{s} .
\end{aligned}
$$




$$
\begin{aligned}
& \mathbf{B}=\nabla \times \mathbf{A}=\left|\begin{array}{ccc}
\hat{x} & \hat{y} & \hat{z} \\
\frac{\partial}{\partial x} & \frac{\partial}{\partial y} & \frac{\partial}{\partial z} \\
A_{x} & A_{y} & A_{z}
\end{array}\right| \\
& =\left(\frac{\partial A_{z}}{\partial y}-\frac{\partial A_{y}}{\partial z}\right) \hat{x}+\left(\frac{\partial A_{x}}{\partial z}-\frac{\partial A_{z}}{\partial x}\right) \hat{y}+\left(\frac{\partial A_{y}}{\partial x}-\frac{\partial A_{x}}{\partial y}\right) \hat{z} . \\
& \mathbf{A} \times(\mathbf{B} \times \mathbf{C})=\mathbf{B}(\mathbf{A} \cdot \mathbf{C})-\mathbf{C}(\mathbf{A} \cdot \mathbf{B}) \\
& \frac{d}{d x} J_{p}(\alpha x)=\alpha P_{p-1}(\alpha x)-\frac{p}{x} J_{p}(\alpha x), \\
& \frac{d}{d x} J_{p}(\alpha x)=-\alpha P_{p+1}(\alpha x)+\frac{p}{x} J_{p}(\alpha x), \\
& \frac{d}{d x} J_{p}(\alpha x)=\frac{\alpha}{2}\left(P_{p-1}(\alpha x)-P_{p+1}(\alpha x)\right), \\
& \cos (a \pm b)=\cos a \cos b \mp \sin a \sin b, \\
& \sin (a \pm b)=\sin a \cos b \pm \cos a \sin b . \\
& \int_{-\lambda / 2 \beta c}^{\lambda / 2 \beta c} \sin \left(\omega_{110}\left(t-\frac{z}{\beta c}\right)\right) d t=-\frac{1}{\omega}\left(\cos \left(\frac{\omega \lambda}{2 \beta c}-\frac{\omega z}{\beta c}\right)-\cos \left(\frac{\omega \lambda}{2 \beta c}+\frac{\omega z}{\beta c}\right)\right), \\
& =-\frac{2}{\omega} \sin \left(\frac{\omega \lambda}{2 \beta c}\right) \sin \left(\frac{\omega z}{\beta c}\right), \\
& \int_{-\lambda / 2 \beta c}^{\lambda / 2 \beta c} \cos \left(\omega_{110}\left(t-\frac{z}{\beta c}\right)\right) d t=\frac{1}{\omega}\left(\sin \left(\frac{\omega \lambda}{2 \beta c}-\frac{\omega z}{\beta c}\right)+\sin \left(\frac{\omega \lambda}{2 \beta c}+\frac{\omega z}{\beta c}\right)\right), \\
& =\frac{2}{\omega} \sin \left(\frac{\omega \lambda}{2 \beta c}\right) \cos \left(\frac{\omega z}{\beta c}\right) \text {. }
\end{aligned}
$$


